\title{
Photosensitivity of the Er/Yb-Codoped Schott IOG1 Phosphate Glass Using 248 nm, Femtosecond, and Picosecond Laser Radiation
}

\author{
Stavros Pissadakis and Irini Michelakaki \\ Institute of Electronic Structure and Laser, Foundation for Research and Technology-Hellas, N. Plastira 100, Vasilika Vouton, \\ P.O. Box 1527, 71110 Heraklion, Greece \\ Correspondence should be addressed to Stavros Pissadakis, pissas@iesl.forth.gr
}

Received 13 August 2008; Accepted 17 September 2008

Recommended by Jacques Albert

\begin{abstract}
The effect of $248 \mathrm{~nm}$ laser radiation, with pulse duration of 5 picoseconds, 500 femtoseconds, and 120 femtoseconds, on the optical properties and the Knoop hardness of a commercial Er/Yb-codoped phosphate glass is presented here. Refractive index changes of the order of few parts of $10^{-4}$ are correlated with optical absorption centers induced in the glass volume, using Kramers-Kroning relationship. Accordingly, substantially lower refractive index changes are measured in volume Bragg gratings inscribed in the glass, indicating that, in addition to the optical density changes, volume dilation changes of negative sign may also be associated with the $248 \mathrm{~nm}$ ultrafast irradiation. The Knoop hardness experimental results reveal that the glass matrix undergoes an observable initial hardening and then a reversing softening and volume dilation process for modest accumulated energy doses, where the Knoop hardness follows a nonmonotonic trend. Comparative results on the Knoop hardness trend are also presented for the case of $193 \mathrm{~nm}$ excimer laser radiation. The above findings denote that the positive or negative evolution of refractive index changes induced by the $248 \mathrm{~nm}$ ultrafast radiation in the glass is dominated by the counteraction of the color center formation and the volume modification effects.
\end{abstract}

Copyright (C) 2008 S. Pissadakis and I. Michelakaki. This is an open access article distributed under the Creative Commons Attribution License, which permits unrestricted use, distribution, and reproduction in any medium, provided the original work is properly cited.

\section{Introduction}

Phosphate glasses are promising host matrixes for the development of free-space and waveguiding optical devices, serving a diverse number of photonic applications. The pentavalent phosphorus can build flexible tetrahedra, where a double oxygen bond occupies one coordination corner, allowing the easy accommodation of other network modifiers, without affecting the glass polymerization chains. That microcoordination structure of the fundamental phosphoric glass matrix leads to specific macroscopic and spectroscopic properties, such as high solubility of rare earths without increasing quenching problems for laser excitation, low transformation temperatures, and potentially high transmissions in the deep ultraviolet wavelengths. Phosphate glasses have been employed as hosts for efficient waveguide [1] and fiber amplifiers [2] and lasers [3], biosensing devices [4], while recently, they have been used as platforms for inscribing into $2 \mathrm{D}$ photonic crystal structures [5]. Even though the above unique optical properties that phosphate glasses exhibit and the numerous applications serve, their photosensitivity has been examined in depth, only recently. The first studies on the phosphate glasses photosensitivity were carried out using femtosecond infrared [6] and near ultraviolet laser sources [7], in pristine or silver-doped matrixes. Later, other studies were presented including deep ultraviolet lasers, by means of $248 \mathrm{~nm}$ [8] and $193 \mathrm{~nm}$ [9] nanosecond excimer and $213 \mathrm{~nm}, 150$ picoseconds Nd:YAG laser radiation [10]. In general, phosphate glasses appear to be of low photosensitivity with the index changes induced in those within the range of $10^{-4}$ and $10^{-5}$, while utilizing high intensities and rather prolonged exposures. Addition of codopand ions, such as Ag [8] or Ge [11], can substantially increase the photosensitivity and 


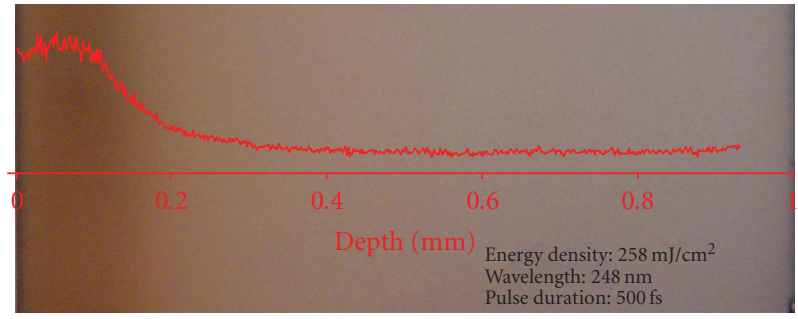

Figure 1: Optical microscope picture obtained from a side polished IOG1 phosphate glass sample, exposed using $248 \mathrm{~nm}, 500$ femtosecond laser radiation and 36000 pulses of $258 \mathrm{~mJ} / \mathrm{cm}^{2}$ energy density. The red line is a normalized gray scale absorption line obtained using image processing.

refractive index changes obtained by more than one order of magnitude. Moreover, the index changes induced in pristine glasses can be either of positive or negative sign, depending upon the exact composition of the exposed glass, the intensities, and energy densities used, as well as the accumulated energy doses dissipated in the glass volume $[9,12]$. The origin of that interesting index engineering behavior is possibly associated with counteracting, radiation triggered, physical mechanisms. In particular, recent studies have revealed that in addition to the strong photochromic effects that occur in phosphate glasses under deep ultraviolet laser irradiation, significant volume modifications also take place, promoted by significant microstructural rearrangements undergone in the glass coordination $[5$, 9].

Herein, we investigate the photosensitivity of the highperformance rare earth doped IOG1 phosphate glass (manufactured by Schott, NY, USA) [13], after exposure to $248 \mathrm{~nm}$, 5-picosecond, 500-femtosecond, and 120-femtosecond laser radiation. The IOG1 glass is optimized for being ionexchangeable using $\mathrm{K}^{+}$and $\mathrm{Ag}^{+}$ions, for developing low loss, high-power lasers, and amplifiers [14]. Our target is to provide and correlate the laser-induced changes in the optical and structural data, for gaining a better insight in the underlying photosensitivity behavior of a commercial ultraphosphate glass matrix. The first part of the study presented herein investigates the effect of the exposure to such high photon energy and extreme intensity radiation on fundamental optical quantities of the glass such as that of the optical absorption and the refractive index. Moreover, the last part of the current study will include additional results on the effect of the irradiation on the Knoop hardness of the pristine and exposed glass.

\section{Experimental}

The IOG1 samples exposed here had a thickness of $1 \mathrm{~mm}$ and contained $2.3 \%$ wt. $\mathrm{Er}_{2} \mathrm{O}_{3}$ and $3.6 \%$ wt. $\mathrm{Yb}_{2} \mathrm{O}_{3}$. Energy dispersive $\mathrm{x}$-ray (EDX) microanalysis measurements performed in similar IOG1 glass batches revealed the existence of other oxides such as $\mathrm{Al}_{2} \mathrm{O}_{3}, \mathrm{La}_{2} \mathrm{O}_{3}$, and $\mathrm{Na}_{2} \mathrm{O}$ [8]. The laser used for the exposures was $248 \mathrm{~nm}$, hybrid LambdaPhysik laser emitting 5-picosecond and 500-femtoseconds

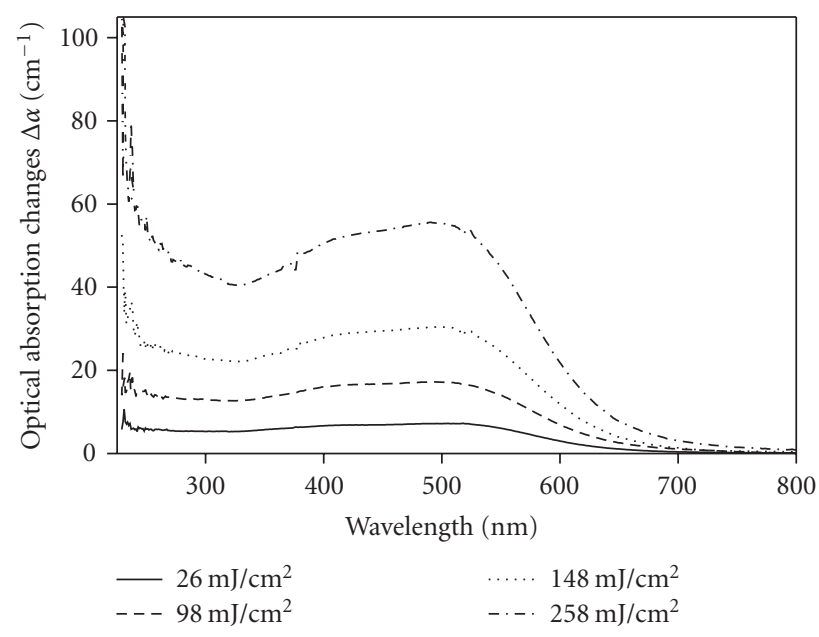

FIGURE 2: Optical absorption changes $\Delta \alpha$ for IOG1 glass samples exposed to different energy densities using 36000 pulses of 500femtosecond pulse duration. The absorption data have been normalized to coloration-damage depth as that was measured using optical microscopy imaging.

pulses, at a repetition rate of $10 \mathrm{~Hz}$. The flux contrast ratio between the emitted picosecond/femtosecond pulse and the nanosecond tail is greater than $95 \%$. For obtaining 120 femtosecond pulses, the 500-femtosecond output was compressed using two reflection gratings which were positioned in parallel, with a spacing of approximately $6 \mathrm{~cm}$ between them [15]. All the exposures performed using 36000 pulses. In the nongrating exposures, the beam was focused onto the sample using a spherical fused silica lens of $20 \mathrm{~cm}$ focal length. A circular aperture was used for selecting the optimum part of the beam, and an oscillatory $\mathrm{CaF}_{2}$ plate was placed in front of the sample to spatially scan the beam for averaging irregularities. The glass samples were exposed to energy densities varying between $25 \mathrm{~mJ} / \mathrm{cm}^{2}$ and $258 \mathrm{~mJ} / \mathrm{cm}^{2}$. The spectrophotometric data were obtained by employing an UV-Visible spectrophotometer, scanning from 190 to $1500 \mathrm{~nm}$.

The spectral data obtained for each sample were normalized to the damage depth induced by the radiation. This volume damage depth was estimated using optical microscopy imaging of the endface of polished samples and subsequent suitable image processing (see Figure 1).

The inscription of Bragg gratings was carried out by employing a $1070 \mathrm{~nm}$ period fused silica, phase mask in "almost" contact mode. The phase mask was of \pm 1 order design, while it was optimized for the $248 \mathrm{~nm}$ wavelength, exhibiting 0 th order less than $1 \%$. For assuring preferential inscription of the $535 \mathrm{~nm}$ period, the phase mask was thoroughly aligned with respect to the planar sample using an external He-Ne red beam. A rectangular aperture was used for selecting the optimum part of the beam and a fused silica $20 \mathrm{~cm}$ cylindrical lens for beam focusing. The samples were exposed to different energy densities which varied between $15 \mathrm{~mJ} / \mathrm{cm}^{2}$ and $\approx 50 \mathrm{~mJ} / \mathrm{cm}^{2}$, for the case 


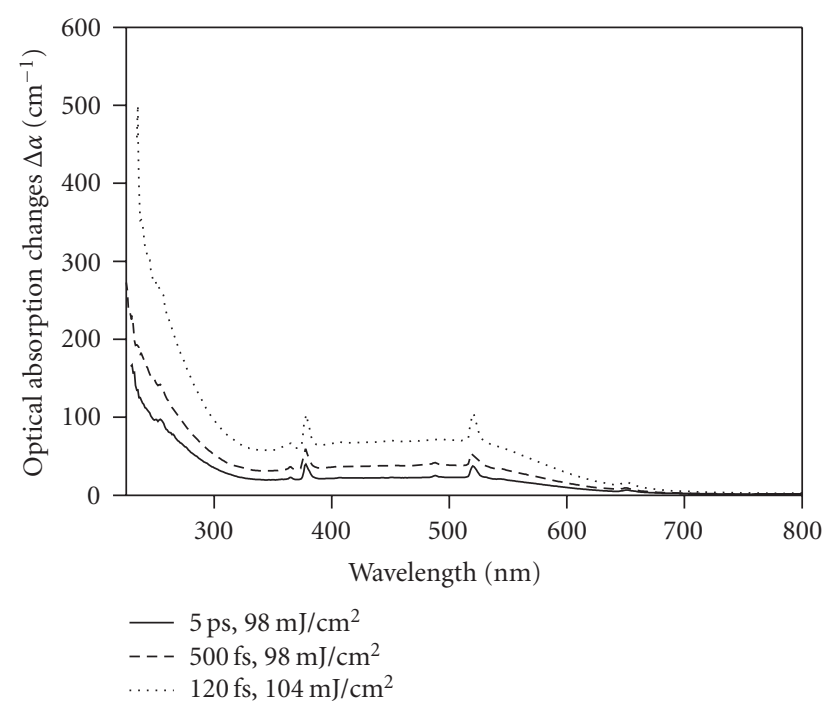

Figure 3: Optical absorption changes $\Delta \alpha$ for IOG1 glass samples exposed with different pulse durations using 36000 pulses and similar energy density.

of 500 -femto second pulse duration. The volume gratings inscribed in the IOG1 glass were measured using a simple diffraction efficiency setup, employing a $632 \mathrm{~nm}$ He-Ne laser, while the gratings probed at Bragg angle. The refractive index changes induced in the glass were evaluated using standard coupled-mode theory for thick, phase-absorption gratings [16], considering a spatial coherence length of the order of the laser beam of $\approx 75 \mu \mathrm{m}$ [17]. Knoop hardness measurements were performed using Matsuzawa, MXT70, digital microhardness indentation microscope, and applying a $50 \mathrm{gf}$ load for 20 -second indentation time. For reducing statistical errors, more than 15 indentations were applied on each sample examined. The exposed sample area was in general wider than $2 \mathrm{~mm}^{2}$, allowing easy accommodation of the indentation traces. The Knoop hardness measurements performed in the pristine sample leaded to a hardness value of 381 , a figure that is very close to the value that is provided by the manufacturer [13]. All spectral, refractive index, and hardness measurements were obtained a week after the laser exposures performed, for allowing defect and structural relaxations to reach a plateau state.

\section{Results and Discussion}

The changes in the optical absorption changes $\Delta \alpha$ of the exposed glass slabs for exposures of different energy densities using 500-femtosecond pulse duration, normalized to the penetration depth of the laser radiation at the 1/e target point, are presented in Figure 2. The exposures were performed using 36000 pulses, while real time probing of the defect $540 \mathrm{~nm}$ band by employing a $2 \mathrm{~mW} 541 \mathrm{~nm}$ HeNe revealed that color center saturation was easily reached shortly after the first 20 minutes of the exposure time, for all the pulse durations and energy densities used.

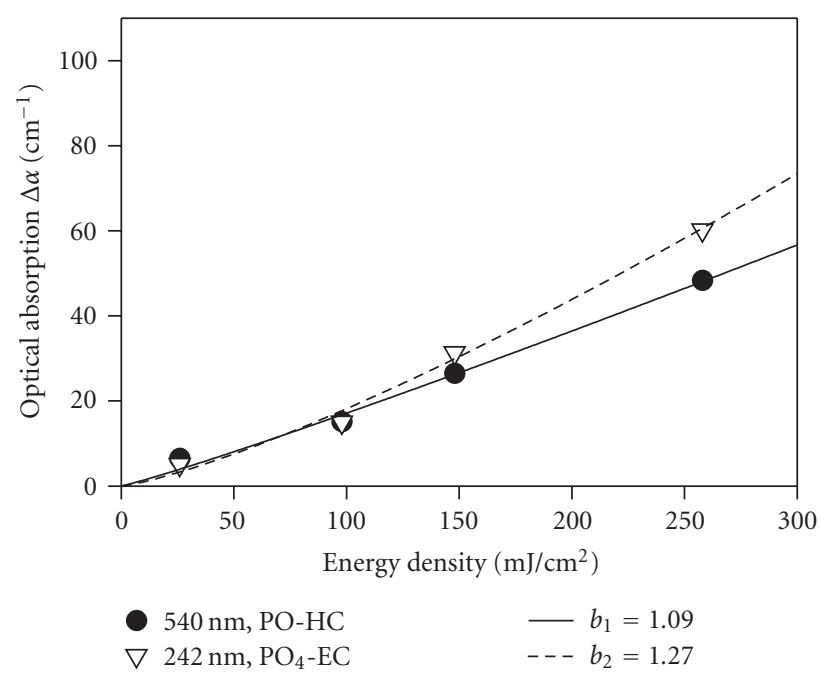

Figure 4: Spectral strength of the PO hole center (HC) located at $540 \mathrm{~nm}$ and that of the $\mathrm{PO}_{4}$ electron center (EC) located at $242 \mathrm{~nm}$ versus energy density, for exposures using $248 \mathrm{~nm}, 500-$ femtosecond laser radiation.

Also, the optical absorption changes $\Delta \alpha$ of the exposed glass for fixed energy density and variable pulse duration are presented in Figure 3. A significant absorption band related to PO bond hole center defects is formed between $220 \mathrm{~nm}$ and $700 \mathrm{~nm}$. The spectral strength of the data presented (see Figure 1) denotes the occurrence of significant electronic changes induced into the exposed glass, by means of cleaving/deforming of the characteristic PO bond and the generation of PO-related centers by electron or hole retrapping [18].

The changes in optical absorption induced by $248 \mathrm{~nm}$, 120 -femtosecond, and 500-femtosecond laser radiation are substantially greater than that obtained using nanosecond pulses of similar energy density [8]; and on the same magnitude with those induced by $193 \mathrm{~nm}$ excimer laser radiation [9]. A point of disagreement with previous reports refers to the bleaching band located at the spectral vicinity of $240 \mathrm{~nm}$ [8]. In the spectral data presented in Figure 2, such bleaching feature does not exist. This may be attributed either to stoichiometric variations of the composition between the IOG1 samples exposed in different studies or to fundamental differences emerge from the underlying photosensitivity mechanism. We speculate that a bleaching band may be formed at that spectral location due to the annihilation of the $\mathrm{PO}_{4}$ electron center; however, its strength can be screened by extensive electron retrapping generated by the longer wavelength bands [18]. Moreover, the accumulated energy density figures needed for inducing such optical density changes, using ultrafast $248 \mathrm{~nm}$ radiation, are significantly lower compared to that for corresponding exposures using $193 \mathrm{~nm}$ or $248 \mathrm{~nm}$ nanosecond radiation [9]. Another comment related to the high durability is that the phosphate glasses exhibit to the ultraviolet radiation. Extreme intensities of the order of $\approx 0.87 \mathrm{TW} / \mathrm{cm}^{2}\left(104 \mathrm{~mJ} / \mathrm{cm}^{2}\right.$ at 120 femtoseconds $)$ can be dissipated inside the glass matrix without triggering 


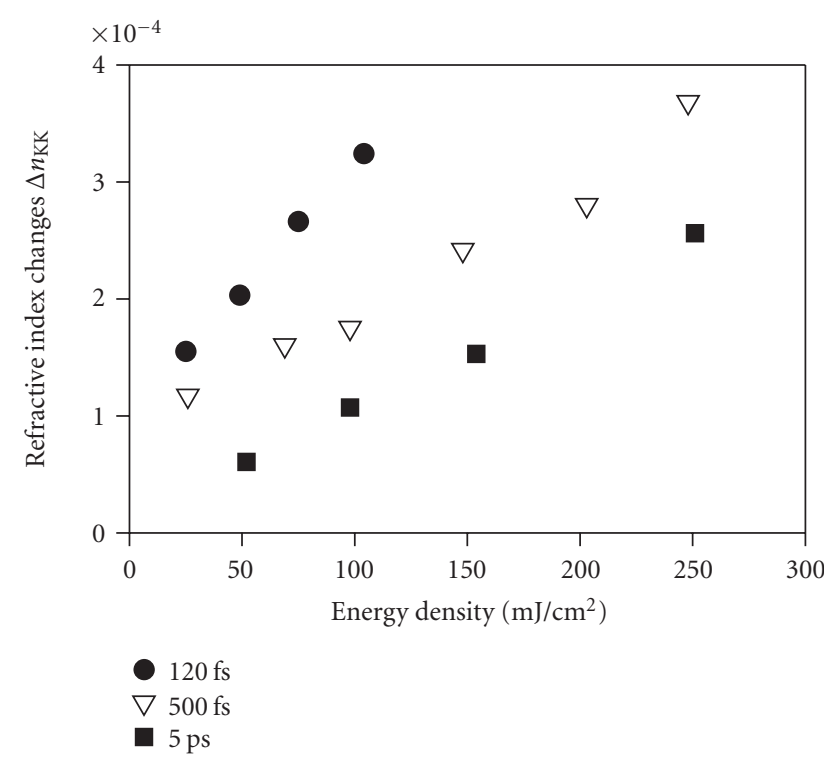

FIGURE 5: Refractive index changes $\Delta n_{\mathrm{KK}}$ induced in the IOG1 phosphate glass using $248 \mathrm{~nm}$, 5-picosecond, 500-femtosecond, and 120 -femtosecond laser radiation versus energy density, estimated using Kramers-Kronig transformation.

surface ablation or filamentation in the glass volume, while the corresponding figure for high-purity silicate glass is $\approx 0.2 \mathrm{TW} / \mathrm{cm}^{2}\left(24 \mathrm{~mJ} / \mathrm{cm}^{2}\right.$ at 120 femtoseconds $)$.

The dependence of the radiation-induced optical density changes $\Delta \alpha$ upon the energy density of the exposure is presented in Figure 4. The data presented refer to two characteristic defect peaks that of $\mathrm{PO}$ hole center (HC) located at $540 \mathrm{~nm}$ and that of the $\mathrm{PO}_{4}$ electron center (EC) located at $242 \mathrm{~nm}[18]$.

Both peaks increase with respect to the energy density of the exposure, while by fitting them to a simple exponential law of the form $\Delta \alpha \propto F^{b}$, where $F$ is the exposure energy density, the $b$ factor is evaluated to be slightly greater than unity (see $b$-data in Figure 4 ). The last indicates that the underlying color center generation process is linearly dependent upon the number of photons per area provided in the glass matrix.

Further, refractive index changes induced by the femtosecond radiation into the glass were estimated by using Kramers-Kronig transformation, from relaxed spectrophotometric data, for the wavelength of $633 \mathrm{~nm}$. The refractive index differences $\Delta n_{\mathrm{KK}}$, which are solely associated with color-centers formation (see Figure 5), were estimated to be of the order of few parts of $10^{-4}$, for all the pulse durations and the energy densities employed. These refractive index changes were calculated using the volume damage depths that were measured using optical microscopy imaging. For reference, the FWHM of these volume damage depths was varied between $300 \mu \mathrm{m}$ for the $26 \mathrm{~mJ} / \mathrm{cm}^{2}$ exposure and $135 \mu \mathrm{m}$ for the $258 \mathrm{~mJ} / \mathrm{cm}^{2}$ exposure, for the case of the 500 -femtosecond radiation (see Figure 1). Accordingly, longer penetration depths up to $1 \mathrm{~mm}$ were measured for the case of the 5-picosecond

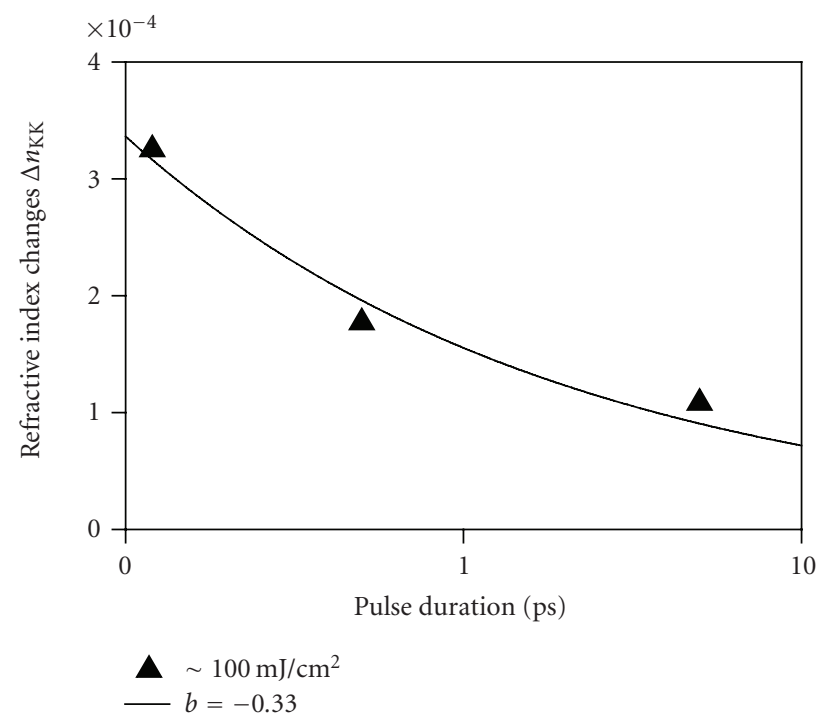

FIGURE 6: Refractive index changes $\Delta n_{\mathrm{KK}}$ induced in the IOG1 phosphate glass versus pulse duration for fixed energy density dose of $248 \mathrm{~nm}$ laser radiation.

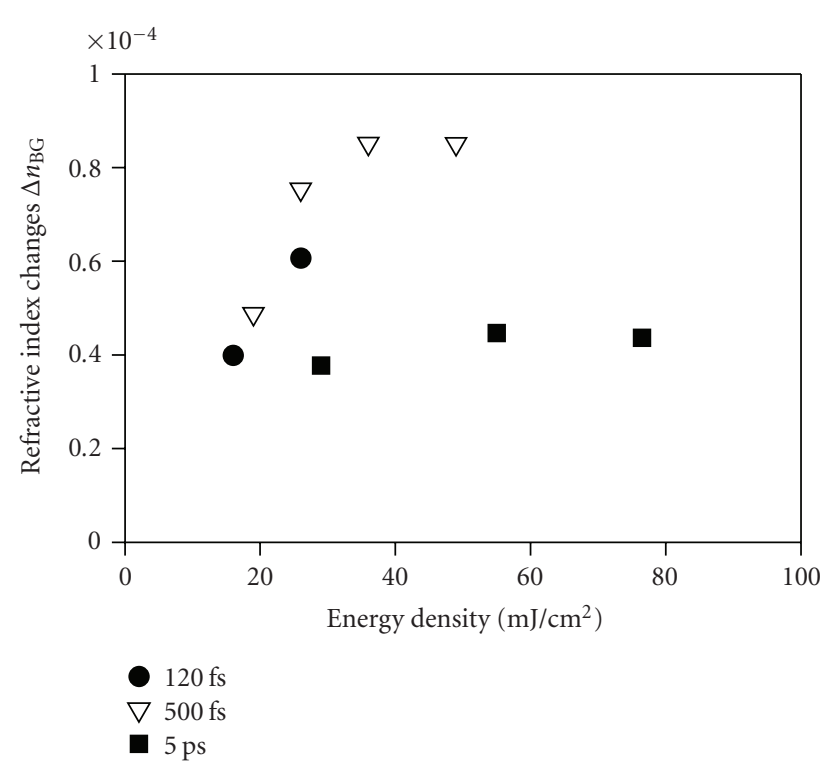

FIGURE 7: Refractive index changes $\Delta n_{\mathrm{BG}}$ measured in Bragg gratings inscribed in the IOG1 glass using diffraction efficiency measurements.

exposures, while these depth figures become smaller for the case of 120 -femtosecond irradiation $(\approx 100 \mu \mathrm{m})$. Similarly, with the spectral strength data presented in Figure 4, the refractive index changes $\Delta n_{\mathrm{KK}}$ of Figure 5 exhibit a linear dependence upon the laser energy density, confirming that the color center generation process and the mutuallyrelated refractive index changes are possible single photon absorption products.

Also, the intensities dissipated inside the glass volume affect the absorption correlated refractive index changes $\Delta n_{\mathrm{KK}}$, where the 120 -femtosecond exposures progress these refractive index changes in a faster rate. However, this 


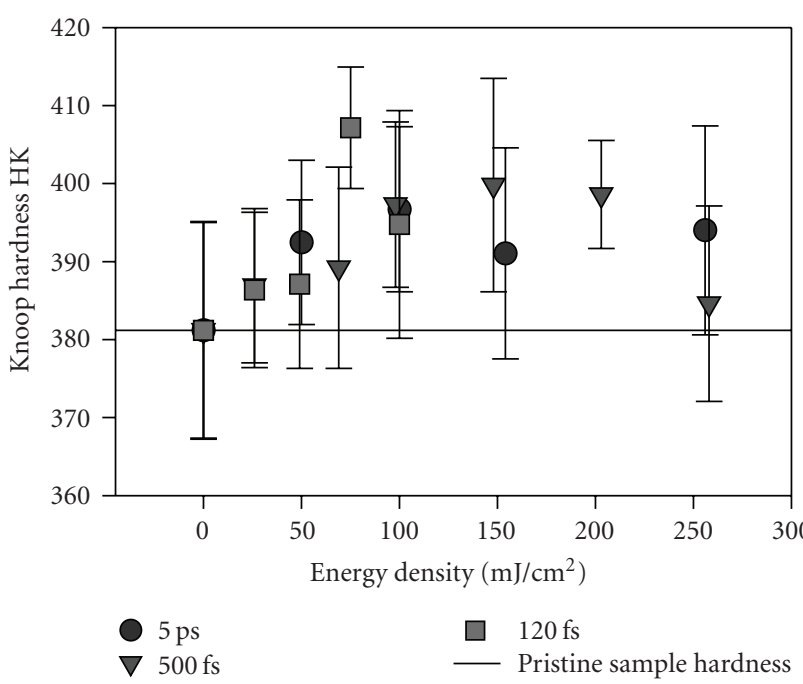

(a)

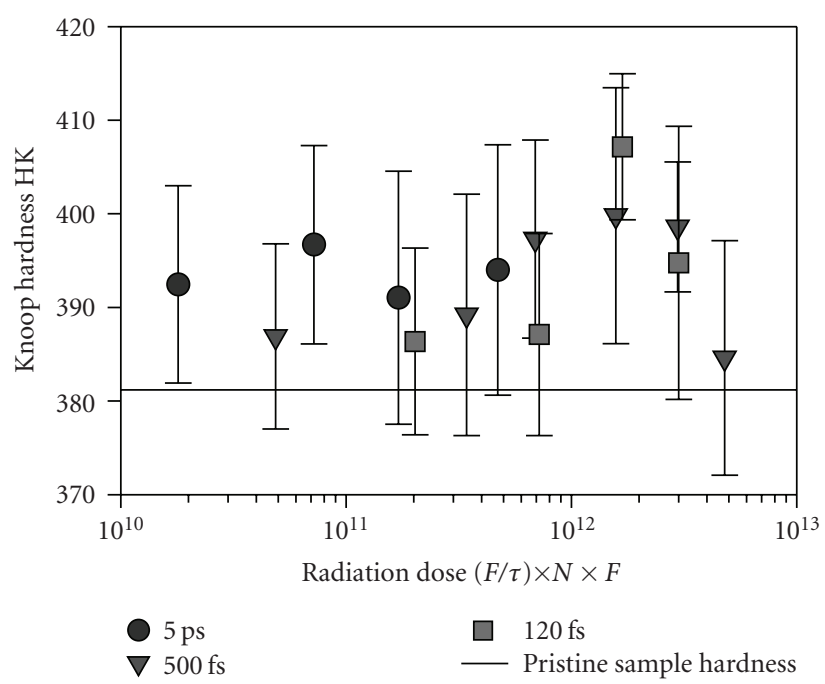

(b)

FIGURE 8: Knoop hardness measurements in pristine and exposed IOG1 phosphate glass versus (a) energy density and (b) energy dose for exposures using 5-picosecond, 500-femtosecond, and 120-femtosecond $248 \mathrm{~nm}$ laser radiation. F: energy density; $\tau$ : pulse duration; $N$ : number of pulses.

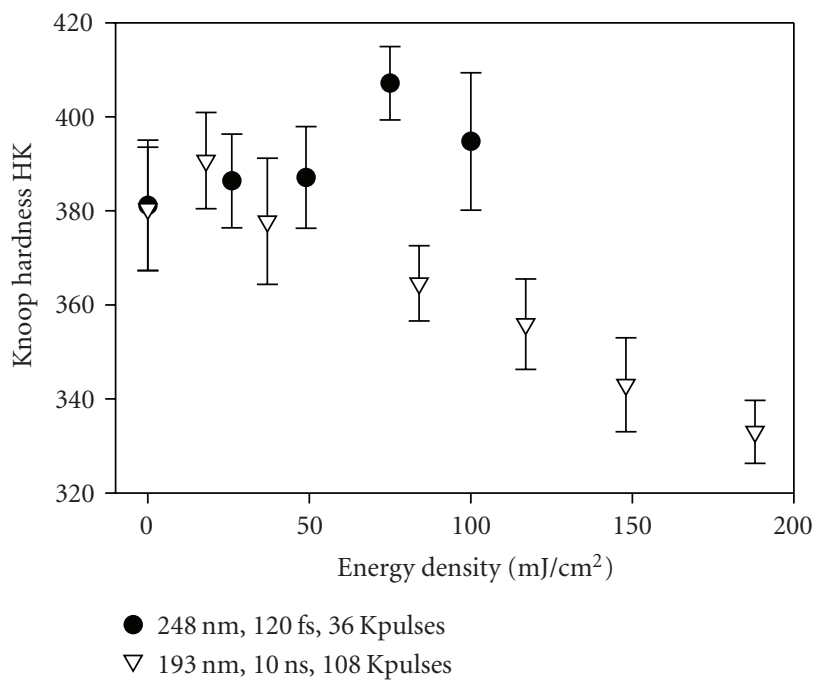

(a)

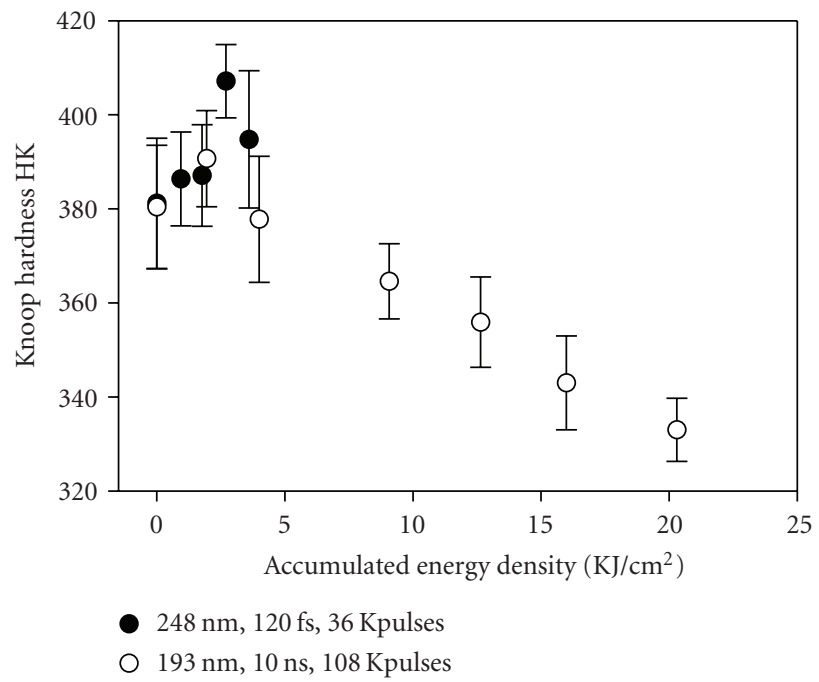

(b)

FIGURE 9: Knoop hardness measurements for IOG1 phosphate glass exposed using 120-femtosecond, $248 \mathrm{~nm}$ and $10 \mathrm{nanoseconds,} 193 \mathrm{~nm}$ laser radiation versus (a) the energy density; and (b) the accumulated energy density of the exposure.

effect may be related with a reduction of the penetration depth due to extensive damage accumulation close to the glass surface vicinity, for greater exposure intensities applied.

In such a case, the photosensitivity products (optical defect concentration or refractive index changes) obtained will exhibit a sublinear dependence upon intensity, reducing the order of interaction below that of a single quantum for the $248 \mathrm{~nm}$ wavelength (see Figure 6).

Additional refractive index change measurements were carried out for Bragg gratings recorded in the $\mathrm{Er} / \mathrm{Yb}$-codoped IOG1 glass matrix (see Figure 7). The refractive index changes $\Delta n_{\mathrm{BG}}$ obtained from diffraction efficiency measurements (Figure 7) are substantially lower than those estimated using Kramers-Kronig transformation, for exposures of the same energy density, while they exhibit a clear saturation trend. The above observation related to the absolute refractive index change magnitude indicates that an additional structural mechanism also contributes to the overall index changes measured in the grating structures. Yliniemi et al. reported that negative refractive index changes dominate the photosensitivity of the same glass using $193 \mathrm{~nm}$ excimer radiation [9], while we have found that similar negative refractive index changes may also occur for exposures using 
$213 \mathrm{~nm}, 150$-picosecond radiation [10]. We believe that a negative refractive index change component also exists in the photosensitivity of the IOG1 phosphate glass using $248 \mathrm{~nm}$ picosecond and femtosecond-laser radiation. This negative sign refractive index component, that was revealed using the above correlated measurements (see Figures 5 and 7), may be associated with either localized stresses in the grating fringes [19] or absolute volume dilation.

For further identifying volume changes induced by the $248 \mathrm{~nm}$ laser irradiation into the IOG1 glass, we have performed Knoop hardness measurements on the exposed samples (see Figures 8(a) and 8(b)). The data of Figures 8(a) and 8 (b) exhibit a nonmonotonous trend, for the three pulse durations employed.

In particular, for the cases of 500-femtosecond and 120femtosecond irradiations, the Knoop hardness of the exposed samples undergoes an initial increase reaching a maximum value that is $\approx 6.8 \%$ greater than that of the pristine glass; while for increased doses [20] (see Figure 8(b)), the absolute hardness follows a declining trend, restoring to normal values. While the Knoop hardness changes induced using 5-picosecond pulses are rather noisy without following a clear trend for the exposures conditions applied, the results referring to the 500-femtosecond and 120-femtosecond pulse durations reach maximum for the same energy dose, indicating that the mechanisms triggered are of the same nature and strength. Changes in the hardness of the glass matrix are directly associated with elastic modulus changes and, therefore, radiation induced volume modifications $[21,22]$. The nonmonotonous increase of Knoop hardness observed here can be initially attributed to volume compaction effects, which may related with the exhausting of a specific type of defect or with the passivation of surface singularities [23]. Accordingly, we believe that the decrease of hardness is related with the glass depolymerization proposed by Yliniemi et al. and observed in exposures using $193 \mathrm{~nm}$ nanosecond excimer laser radiation [9]. We have also performed micro-Raman spectroscopy using a $473 \mathrm{~nm}$ Ar-ion laser, in glass samples exposed using 120 femtoseconds, $100 \mathrm{~mJ} / \mathrm{cm}^{2}$, where the measurements show $\mathrm{a} \approx 2.5 \%$ broadening of the two fundamental peaks located at $704 \mathrm{~cm}^{-1}$ (POP symmetric bond) and $1184 \mathrm{~cm}^{-1}\left(\mathrm{PO}_{2}\right.$ symmetric bond), compared to that of the pristine glass; denoting the generation of a broad band of structural defects. Therefore, a glass depolymerization model based on the extensive cleaving of the fundamental PO bond may be used for explaining the volume dilation effects observed in the case of this ultraphosphate glass, when exposed to $248 \mathrm{~nm}$ picosecond and femtosecond radiations.

We believe that longer exposures employing $248 \mathrm{~nm}$ femtosecond radiation will succeed in lowering the Knoop hardness below the levels of the pristine sample. Such massive reduction of the surface hardness and the glass density has been observed in exposures using $193 \mathrm{~nm}$ excimer laser radiation (see Figures $9(\mathrm{a})$ and $9(\mathrm{~b})$ ), where the greater energy per photon progresses such reverse sign, structural, and volume modification effects much faster than the $248 \mathrm{~nm}$ radiation. These significant structural changes are induced for the case of $193 \mathrm{~nm}$ radiation without employing extreme sub-TW $/ \mathrm{cm}^{2}$ intensities, but by providing a photon energy that is close or slightly higher than the bandgap of the exposed material, while utilizing $\mathrm{MW} / \mathrm{cm}^{2}$ intensities. However, the massive decrease of the Knoop hardness and the accompanying strong volume dilation effects induced by the $193 \mathrm{~nm}$ exposures are observed in expense of increased accumulated energy densities; which in turn are manifold larger than those utilized for the $248 \mathrm{~nm}, 120$-femtosecond irradiations (see Figure 9(b)). Finally, in Figure 9(b), we see that the Knoop hardness modifications induced by both wavelengths follow rather similar trends, while peaking at quite similar accumulated energy density value. Further work on the estimation of the elastic modulus of the glass exposed using $193 \mathrm{~nm}$ laser radiation and its correlation with the Knoop hardness and the exposure conditions are presented in detail elsewhere [24].

\section{Conclusions}

The photosensitivity of the Er/Yb-codoped IOG1 phosphate glass using $248 \mathrm{~nm}$, 5-picosecond, 500-femtosecond, and 120 -femtosecond laser radiation is presented here. We employed spectrophotometric measurements for identifying the absorption bands induced by the radiation; while later on, these spectral measurements were interpreted using Kramers-Kroning relationship for obtaining refractive index changes associated with this specific mechanism. The refractive index changes correlated with optical absorption centers induced in the glass volume are of the order of few parts of $10^{-4}$. Moreover, we employed Bragg grating recording in the glass volume for estimating the overall refractive index changes induced by the ultrafast radiation exposures. These measurements revealed that the overall refractive index changes formed are of substantially lower magnitude than those estimated using the Kramers-Kroning approach, indicating that, in addition to the optical density changes, volume dilation changes of negative sign are also associated with the $248 \mathrm{~nm}$ ultrafast irradiation. The Knoop hardness microindentation experimental results obtained show that the exposed glass matrix undergoes an observable initial hardening and then a reversing softening and volume dilation process for modest accumulated energy doses, wherein the Knoop hardness follows a nonmonotonic trend. Such observation justifies the lower refractive index changes gratings probed in volume Bragg grating reflectors, where the positive refractive index changes, due to color centers, are competed by the negative index changes induced by the volume dilation effects. We are working on a deeper investigation of the photosensitivity of phosphate glasses using ultraviolet nanosecond and femtosecond laser radiation, and the correlation of the results with those obtained for other well-studied glasses (i.e., Silicate and Germanosilicate amorphous matrices), using the same wavelengths and pulse durations. Also, gaining a better understanding on the volume damage and modification effects can be further exploited in the high yield selective chemical etching micro/nanoprocessing of the specific glass [5]. 


\section{Acknowledgments}

Stavros Pissadakis gratefully acknowledges Leo Gilroy (Schott, NY, USA) for kindly providing the glass samples, as well as Vicky Vamvakaki and Nikos Chaniotakis (Laboratory of Analytical Chemistry, Chemistry Department, University of Crete) for performing the micro-Raman measurements.

\section{References}

[1] D. L. Veasey, D. S. Funk, P. M. Peters, et al., "Yb/Er-codoped and $\mathrm{Yb}$-doped waveguide lasers in phosphate glass," Journal of Non-Crystalline Solids, vol. 263-264, pp. 369-381, 2000.

[2] B. C. Hwang, S. Jiang, T. Luo, et al., "Erbium-doped phosphate glass fibre amplifiers with gain per unit length of $2.1 \mathrm{~dB} / \mathrm{cm}$," Electronics Letters, vol. 35, no. 12, pp. 1007-1009, 1999.

[3] J. Albert, A. Schüzgen, V. L. Temyanko, S. Honkanen, and N. Peyghambarian, "Strong Bragg gratings in phosphate glass single mode fiber," Applied Physics Letters, vol. 89, no. 10, Article ID 101127, 3 pages, 2006.

[4] M. Krauß and J. Leißner, "Bio-sensors for microbiological atmospheric pollution," in Proceedings of the 7th ESG Conference on Glass Science and Technology, Athens, Greece, April 2004, paper P-EI1.

[5] S. Pissadakis and C. Pappas, "Planar periodic structures fabricated in Er/Yb-codoped phosphate glass using multibeam ultraviolet laser holography," Optics Express, vol. 15, no. 7, pp. 4296-4303, 2007.

[6] J. W. Chan, T. R. Huser, S. H. Risbud, J. S. Hayden, and D. M. Krol, "Waveguide fabrication in phosphate glasses using femtosecond laser pulses," Applied Physics Letters, vol. 82, no. 15, pp. 2371-2373, 2003.

[7] Y. Watanabe, G. Namikawa, T. Onuki, K. Nishio, and T. Tsuchiya, "Photosensitivity in phosphate glass doped with $\mathrm{Ag}^{+}$ upon exposure to near-ultraviolet femtosecond laser pulses," Applied Physics Letters, vol. 78, no. 15, pp. 2125-2127, 2001.

[8] S. Pissadakis, A. Ikiades, P. Hua, A. K. Sheridan, and J. S. Wilkinson, "Photosensitivity of ion-exchanged Er-doped phosphate glass using $248 \mathrm{~nm}$ excimer laser radiation," Optics Express, vol. 12, no. 14, pp. 3131-3136, 2004.

[9] S. Yliniemi, S. Honkanen, A. Ianoul, A. Laronche, and J. Albert, "Photosensitivity and volume gratings in phosphate glasses for rare-earth-doped ion-exchanged optical waveguide lasers," Journal of the Optical Society of America B, vol. 23, no. 12, pp. 2470-2478, 2006.

[10] C. Pappas and S. Pissadakis, "Periodic nanostructuring of $\mathrm{Er} / \mathrm{Yb}$-codoped IOG1 phosphate glass by using ultraviolet laser-assisted selective chemical etching," Journal of Applied Physics, vol. 100, no. 11, Article ID 114308, 6 pages, 2006.

[11] S. Suzuki, A. Schülzgen, S. Sabet, J. V. Moloney, and N. Peyghambarian, "Photosensitivity of Ge-doped phosphate glass to $244 \mathrm{~nm}$ irradiation," Applied Physics Letters, vol. 89, no. 17, Article ID 171913, 3 pages, 2006.

[12] C. Pappas, Fabrication of $1 D$ and $2 D$ relief Bragg reflectors in rare earth doped phosphate glass by employing holographic laser induced structural modification, M.S. thesis, Physics Department, University of Crete, Heraklion, Greece, 2006.

[13] Schott Glass datasheets for IOG-1 glass.

[14] S. Yliniemi, J. Albert, Q. Wang, and S. Honkanen, "UVexposed Bragg gratings for laser applications in silver-sodium ion-exchanged phosphate glass waveguides," Optics Express, vol. 14, no. 7, pp. 2898-2903, 2006.
[15] S. Szatmári and F. P. Schäfer, "Simplified laser system for the generation of $60 \mathrm{fs}$ pulses at $248 \mathrm{~nm}$," Optics Communications, vol. 68, no. 3, pp. 196-202, 1988.

[16] H. M. Smith, Ed., Holographic Recording Materials, Springer, Berlin, Germany, 1977.

[17] S. Kawata, I. Hikima, Y. Ichihara, and S. Watanabe, "Spatial coherence of KrF excimer lasers," Applied Optics, vol. 31, no. 3, pp. 387-396, 1992.

[18] D. Ehrt, P. Ebeling, and U. Nature, "UV transmission and radiation-induced defects in phosphate and fluoridephosphate glasses," Journal of Non-Crystalline Solids, vol. 263264, pp. 240-250, 2000.

[19] H. G. Limberger, P.-Y. Fonjallaz, R. P. Salathé, and F. Cochet, "Compaction- and photoelastic-induced index changes in fiber Bragg gratings," Applied Physics Letters, vol. 68, no. 22, pp. 3069-3071, 1996.

[20] D. C. Allan, C. Smith, N. F. Borrelli, and T. P. Seward III, "193$\mathrm{nm}$ excimer-laser-induced densification of fused silica," Optics Letters, vol. 21, no. 24, pp. 1960-1962, 1996.

[21] Y. Bellouard, T. Colomb, C. Depeursinge, M. Dugan, A. A. Said, and P. Bado, "Nanoindentation and birefringence measurements on fused silica specimen exposed to low-energy femtosecond pulses," Optics Express, vol. 14, no. 18, pp. 83608366, 2006.

[22] D. B. Marshall, T. Noma, and A. G. Evans, "A simple method for determining elastic-modulus-to-hardness ratios using Knoop indentation measurements," Journal of the American Ceramic Society, vol. 65, no. 10, pp. c175-c176, 1982.

[23] W. Meisel, D. Sprenger, and P. Guetlich, "Surface versus bulk composition of a phosphate glass," Surface and Interface Analysis, vol. 22, no. 1-12, pp. 267-270, 1994.

[24] I. Michelakaki and S. Pissadakis, "Atypical behaviour of the surface hardness and the elastic modulus of a phosphate glass matrix under $193 \mathrm{~nm}$ laser irradiation," Applied Physics A. In press. 